\title{
Protection pneumatique des barrages contre les surpressions hydrodynamiques d'origine sismique
}

\author{
Pneumatic protection of large dams \\ against hydrodynamic pressure \\ fluctuations of seismic origin
}

\author{
F. Biesel \\ Ingénieur Conseil
}

\section{Nature du problème étudié}

Un barrage de réservoir est soumis à des contraintes statiques résultant de son propre poids, des pression hydrostatiques, de conditions d'ancrage spécifiques etc. Lorsqu'il est soumis à un séisme, des forces d'inertie viennent s'ajouter au poids, des fluctuations de pression hydrodynamiques s'ajoutent aux pressions hydrostatiques et les contraintes dues aux conditions d'ancrage peuvent également être modifiées soit par suite de déformations d'ensemble du site, soit par suite de réactions dynamiques, plus ou moins élastiques, des fondations.

De nombreuses études ont été faites pour tenter d'évaluer les surcharges dynamiques ainsi dues aux séismes. Les plus perfectionnées de ces études tiennent compte, dans toute la mesure du possible, de l'ensemble des phénomènes en jeu et de leurs interactions. La présente étude est beaucoup plus limitée. Elle a pour objet l'évaluation de l'intérêt et de l'efficacité d'un dispositif de protection pneumatique des barrages contre les fluctuations hydrodynamiques. Ce dispositif, couvert par un brevet, consiste à aménager une zone de grande compressibilité couvrant le parement amont du barrage. Cette compressibilité est obtenue à l'aide de poches d'air retenues prisonnières au voisinage de cette paroi. La figure 1 montre schématiquement deux des dispositions possibles pour une telle poche d'air aménagée dans le parement amont. Notons, sur la figure 1a, qu'un diaphragme $\mathrm{D}$ peut introduire un effet amortisseur et une canalisation $C$ un effet d'inertie dans les fluctuations de la poche d'air A. Dans l'étude ci-après, nous négligerons cet effet d'inertie, ce qui signifie que nous admettrons que les poches d'air communiquent avec l'eau du barrage par des passes de grande section et que les amortissements sont réalisés par des plaques perforées ou poreuses également de grande surface, ainsi que cela est montré sur la figure lb.
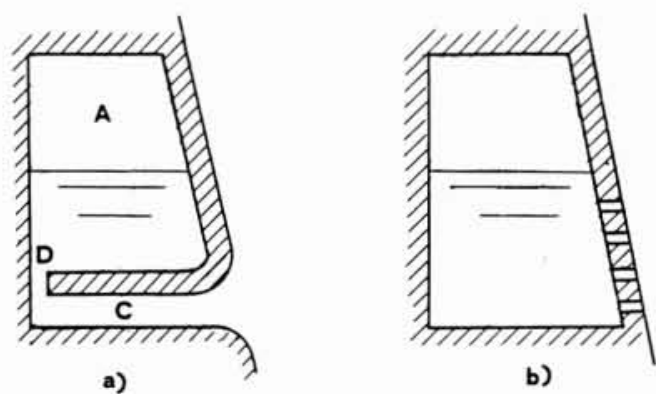

b)

Figure 1 - Disposition schématique de poches d'air dans le parement amont d'un barrage. Le réservoir est à droite pour les deux exemples a et $b$. 


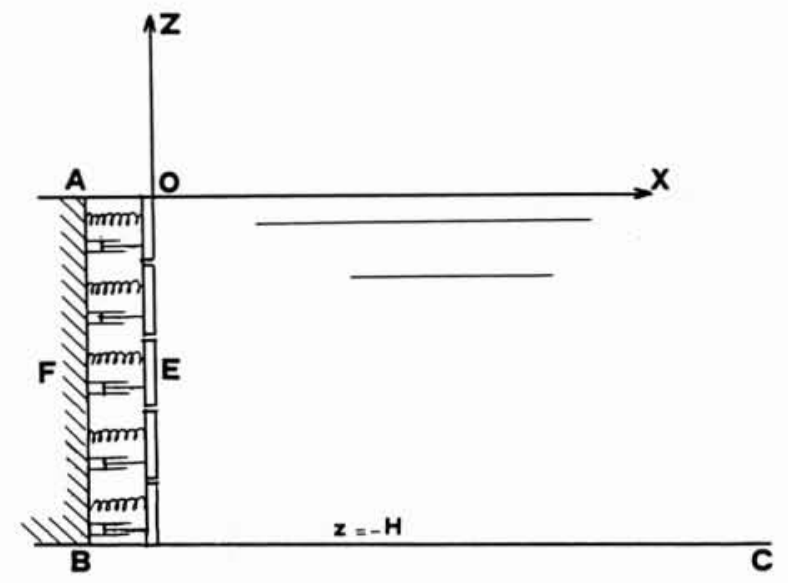

Figure 2 - Schématisation du mécanisme de protection pneumatique et système de coordonnées utilisés dans le calcul.

Si ces poches d'air ne sont pas trop éloignées les unes des autres, leur effet peut être considéré comme " réparti " sur toute la surface du parement. La figure 2 montre un équivalent mécanique de cette situation. Tout se passe comme si le parement E, au contact de l'eau, était relié au corps du barrage F par un " amortisseur " comportant des ressorts de rigidité $\mathrm{r}$ (par unité de surface) et présentant des coefficients de frottement $\mathrm{f}$, également par unité de surface. Les dispositifs réels auront vraisemblablement des lois de frottement et de rigidité non linéaires, c'est le cas de l'exemple schématique montré par la figure 1, nous nous limiterons cependant à des calculs linéarisés. Il est clair, qu'en toute rigueur, nos résultats ne seront valables que pour des perturbations d'ampleur limitée mais, pratiquement, cette limitation ne sera pas gênante avec les ordres de grandeur des séismes que l'on doit raisonnablement prendre en compte. De toute façon, des études complémentaires tenant compte de la non linéarité des amortisseurs seraient très faisables en cas de nécessité.

Les résultats que nous présentons dans cet article sont otbenus sur un cas schématique correspondant à un calcul bidimensionnel $(X-Z)$. Ils se réfèrent à un barrage rectiligne, infiniment long dans la direction de l'axe $O Y$, de profondeur constante $(Z=-H)$ et s'étendant à l'infini vers l'amont, dans la direction de l'axe $O X$. Le parement amont du barrage est vertical (plan $X=0$ ) et le mouvement sismique est une translation en bloc (sans déformation) de l'ensemble du site barrage compris. Les déformations de ce dernier sont donc également négligées.

Les coefficients $r$ et $f$, définis plus haut, du dispositif de protection pneumatique sont les mêmes pour toute la surface du parement amont. Cette option n'est prise que pour simplifier les calculs, elle n'implique aucunement qu'une telle uniformité soit recommandable, au contraire, la répartition de $r$ et $f$ le long du parement est une des caractéristiques qui pourra être optimisée cas par cas. II semble, en particulier, que l'on aura intérêt à faire croitre la rigidité $r$ avec la profondeur.
Nous ne pensons pas que les diverses approximations ainsi consenties soient de nature à fausser les ordres de grandeur relatifs à l'efficacité du dispositif de protection. Par contre, il est apparu nécessaire de ne pas négliger la compressibilité de l'eau puisque, précisément, les phénomènes d'élasticité sont au cœur du problème.

2. Ordre de grandeur relatif des effets sismiques hydrodynamiques

Le dispositif de protection pneumatique n'a aucun effet sur les forces sismiques d'inertie qui agissent sur la masse du barrage lui-même. Il est donc intéressant de se demander si les forces hydrodynamiques sismiques ne sont pas d'un ordre de grandeur sensiblement inférieur, ce qui pourrait annuler l'intérêt de la protection pneumatique.

On peut faire une première estimation de ces ordres de grandeur à partir de la formule empirique de Westergaard qui est très couramment utilisée. Dans le cas d'un barrage poids schématique, de section triangulaire, ayant la hauteur $\mathrm{H}$ de la retenue, une base de longueur $0,8 \mathrm{H}$ et une densité égale à 2,5 fois celle de l'eau, la formule de Westergaard donne les conclusions suivantes.

Pour le barrage entièrement rempli et une accélération sismique horizontale, la poussée sismique de l'eau est environ $60 \%$ de la force d'inertie qui s'exerce sur la masse du barrage (ce chiffre devient $70 \%$ si l'on considère les couples de renversement).

Les poussées hydrodynamiques sismiques ne sont donc absolument pas négligeables même pour un barrage poids.

Dans le cas d'un barrage voûte, le volume de béton est plusieurs fois inférieur et par conséquent les fluctuations de pression hydrodynamiques sont très supérieures aux forces d'inerties de masse.

Il faut ajouter à cela que les forces de pression hydrodynamiques peuvent être réparties de façon beaucoup plus irrégulières que les forces d'inertie, ce qui peut les rendre sensiblement plus dangereuses pour des voûtes relativement minces.

Les études "dynamiques" plus poussées que celle de Westergaard montrent que les pointes de pression hydrodynamiques dépassent assez considérablement celles données par cet auteur. Ce point est d'ailleurs confirmé par les résultats de la présente étude.

En définitive, on peut conclure que les fluctuations de pression hydrodynamiques constituent l'essentiel des surcharges sismiques agissant sur un barrage voûte et environ la moitié de celles agissant sur un barrage poids.

Notons encore que le dispositif de protection pneumatique présente un avantage qui peut paraître un peu académique mais qui a cependant un intérêt pratique certain. En réalisant une sorte de " coupure " mécanique, entre le barrage et l'eau, il rend plus faciles et donc plus sûrs les calculs dynamiques de l'ouvrage. Les mouvements du barrage peuvent être étudiés avec une bonne approximation sans tenir compte de ses interactions complexes avec l'eau et, symétriquement, l'étude des fluctuations de pression hydrodynamiques peut se faire en négligeant les déformations du barrage, ce que nous avons mis à profit dans les calculs de cette note. 


\section{Exemple numérique}

Nous donnons ci-après les résultats d'un calcul exécuté sur un exemple typique et nous renvoyons en annexe le développement des formules utilisées. Le cas traité est celui d'un barrage de 100 mètres (profondeur de l'eau). Les accélérations sismiques horizontale et verticale sont étudiées séparément. Nous avons calculé les valeurs maximales des poussées $P$ et des couples de renversement $C$ dus aux efforts hydrodynamiques et les résultats sont donnés sous la forme $p=\mathrm{P} / \mathrm{Po}$ et $c=\mathrm{C} / \mathrm{Co}$, Po et $\mathrm{Co}$ étant la poussée et le couple correspondant à une accélération verticale constante égale à la valeur maximale de l'accélération sismique. Par exemple, si cette valeur est égale à $g / 10$, Po et Co sont le dixième de la poussée hydrostatique et du couple de renversement hydrostatique.

Les rapports $p$ et $c$ donnent donc une sorte de coefficient de réponse dynamique (majoration ou minoration) de l'effet des séismes par rapport à l'effet d'accélérations constantes ou très lentement variables (pour les séismes verticaux de très basse fréquence, par exemple, ces coefficients deviennent donc très voisins de l'unité).

La figure 3 montre les valeurs de $p$, en fonction de la fréquence pour un séisme horizontal. Le trait plein correspond à un barrage sans protection, le trait pointillé à un barrage avec dispositif de protection mais sans amortissement $(f=0)$ et le trait interrompu à un barrage muni d'un dispositif de protection normal avec amortissement $(f / r=0,25$ c.g.s.). Pour les exemples où il y a un dispositif de protection, son élasticité est celle d'une couche d'air de $10 \mathrm{~cm}$ d'épaisseur à la pression atmosphérique. L'épaisseur "répartie " des poches d'air varie donc de $10 \mathrm{~cm}$ en surface à $110 \mathrm{~cm}$ au fond. Dans les applications réelles, on pourrait certainement se dispenser d'avoir de telles quantités d'air dans les zones profondes, leur raison d'être n'est, rappelons le, que de simplifier les calculs en rendant le coefficient d'élasticité $r$ constant.

On constate que la protection sans amortissement donne un résultat excellent pour les séismes de fréquence relativement élevée mais que, par contre, les séismes de basse fréquence peuvent être considérablement amplifiés. On peut comparer ce cas à celui des balancements d'un véhicule ayant une suspension élastique mais aucun amortisseur.

Avec la valeur d'amortissement choisie pour le troisième cas, on constate que la protection contre les séismes de fréquence relativement élevée (supérieure à 1 hertz) est un peu moins bonne mais reste extrêmement satisfaisante. Par ailleurs, la "résonance " aux basses fréquences est bien amortie et ne se traduit plus que par un léger gonflement de la réponse avec une amplification maximale de l'ordre de 1,4 pour des fréquences de l'ordre de 0,5 hertz.

Les séismes réels sont irréguliers ce qui signifie que l'on peut assimiler leurs mouvements à une superposition de composantes sinusoïdales dont les fréquences s'étendent sur une gamme assez large. Ainsi, la perte sur les composantes lentes sera-t-elle, en général, largement compensée par le gain sur les fréquences élevées. Ceci est d'autant plus intéressant que ces dernières sont les plus "brisantes " ce qui est important pour un matériau relativement fragile comme le béton.

L'effet de la protection ne pourrait être négatif que pour des séismes dont presque toute l'énergie serait concentrée autour d'une fréquence de l'ordre de 0,5 hertz. Il importe de remarquer que ce cas très exceptionnel correspondrait le plus souvent à des tremblements de terre catastrophiques accompagnés de dislocations importantes

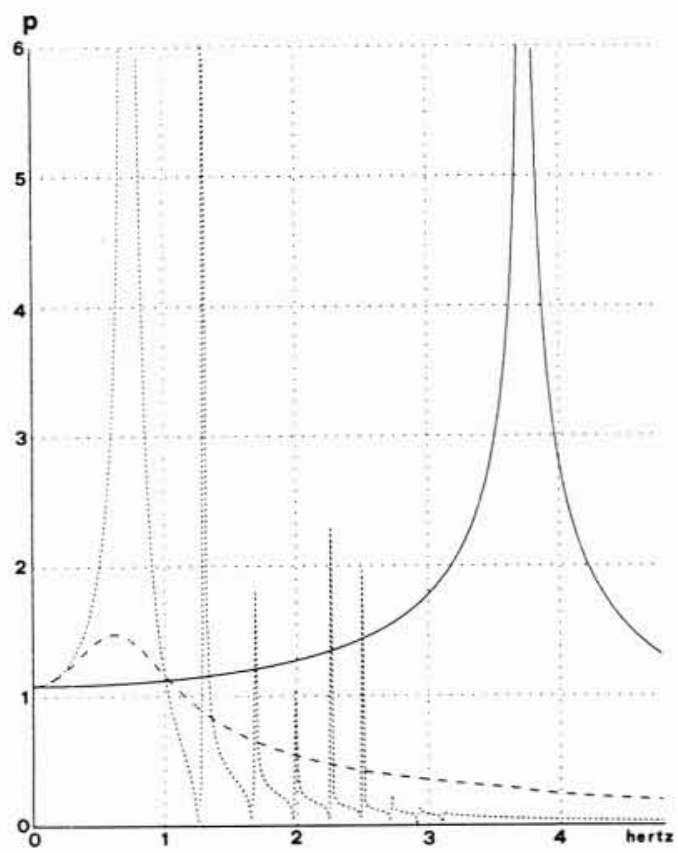

Figure 3 - Variation du coefficient de réponse hydrodynamique $p$ en fonction de la fréquence pour une accélération sismique sinusoîdale horizontale. Le trait plein correspond à un barrage rigide sans protection pneumatique., le pointillé à un barrage muni d'une protection non amortie et le trait interrompu à un barrage muni d'une protection avec amortissement.

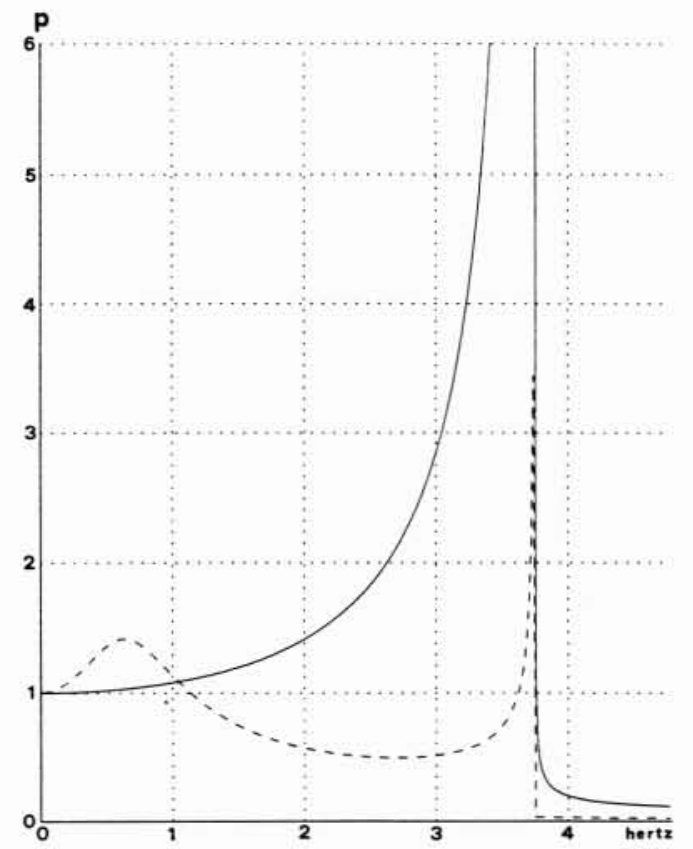

Figure 4 - Variation du coefficient de réponse hydrodynamique $p$ en fonction de la fréquence pour une accélération sismique sinusoïdale verticale. Le trait plein correspond à un barrage rigide sans protection pneumatique et le trait interrompu à un barrage muni d'une protection avec amortissement. 


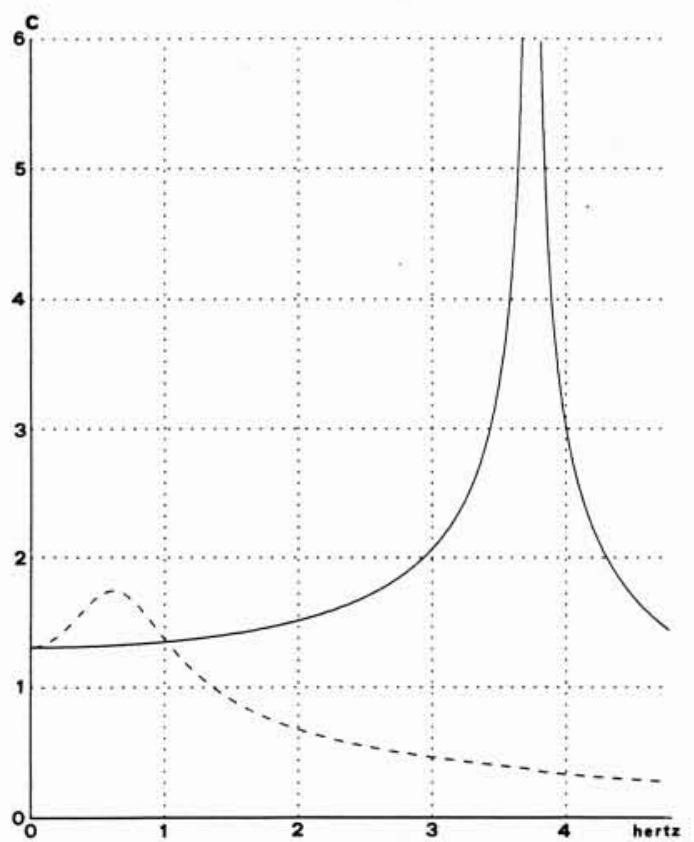

Figure 5 - Variation du coefficient de réponse hydrodynamique $c$ en fonction de la fréquence pour une accélération sismique sinusoïdale horizontale.

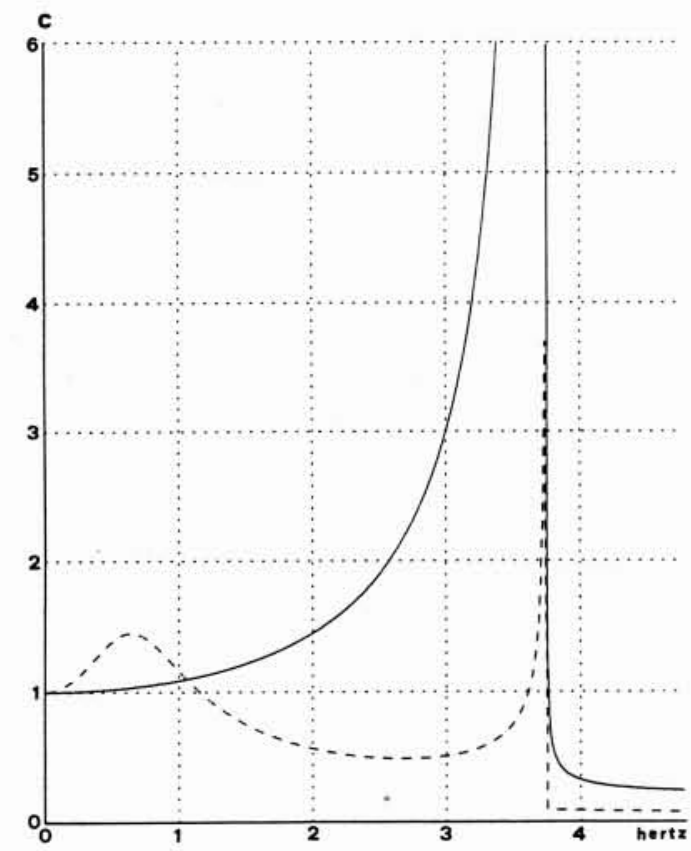

Figure 6 - Variation du coefficient de réponse hydrodynamique $c$ en fonction de la fréquence pour une accélération sismique sinusoïdale verticale. de l'ensemble du site et de l'ouvrage. En effet, même avec ces accélérations maximales de $g / 10$, ce qui correspond à une hypothèse de séismicité modérée, les déplacements seraient de l'ordre de $20 \mathrm{~cm}$ pour une fréquence de 0,5 hertz.

Les figures 4,5 et 6 permettent d'autres comparaisons entre les valeurs des pressions hydrodynamiques avec ou sans protection pneumatique. Ces figures sont présentées comme la précédente, la courbe en traits pleins correspondant au barrage sans protection et la courbe en traits interrompus correspondant à une protection avec amortissement. La figure 4 donne les coefficients $p$ pour des séismes verticaux, les figures 5 et 6 donnent les coefficients $c$ pour des séismes respectivement horizontaux et verticaux.

On voit facilement que ces résultats complémentaires conduisent exactement aux mêmes conclusions d'ensemble. En définitive, la protection pneumatique offre un accroissement considérable de la sécurité des grands barrages pour tous les séismes contre lesquels on peut raisonnablement espérer se prémunir.

Comme ce dispositif de protection peut également s'adjoindre à des barrages existants, il est vraisemblable qu'il soit appelé à devenir aussi banal, dans les zones de séismicité reconnue, que les suspensions élastiques et les amortisseurs le sont devenus pour les véhicules terrestres.

\section{Annexe mathématique}

Dans cette annexe, nous donnons les éléments nécessaires pour reconstituer l'ensemble des formules théoriques qui ont été utilisées dans les exemples numériques.

\subsection{Equation générale et conditions aux limites}

La figure 2 montre le système de coordonnées utilisé. L'origine $O$ est à l'intersection du plan d'eau et du parement amont du barrage dans leur position de repos. Cette intersection est l'axe $O Y$ inutilisé ici. L'axe $O Z$ est ascendant et l'axe $O X$ est dirigé vers l'amont.

Le mouvement sismique est une translation en bloc du sol $B C$ et du corps du barrage $A B$. La loi de ce mouvement est sinusoïdale dans le temps $t$ et de fréquence angulaire $\omega$. On étudie donc séparément chaque fréquence, ce qui permet de reconstituer l'effet de n'importe quelle autre loi de mouvement dans le temps, en passant par une décomposition en série ou en intégrale de Fourier. De plus, nous étudierons aussi séparément les effets des accélérations sismiques verticales et horizontales (parallèles à l'axe $O X)$ qui pourront également être recombinées linéairement si nécessaire.

Pour toutes les quantités dépendant sinusoïdalement du temps, nous adoptons la notation imaginaire classique. Ainsi, par exemple, l'accélération sismique horizontale sera désignée par la constante complexe $\alpha_{0}$, ce qui signifie que sa valeur physique est la partie réelle de

$$
\alpha_{0} \cdot \operatorname{EXP}(j \cdot \omega \cdot t)
$$

$j$ étant l'unité imaginaire. 
La vitesse sismique correspondante sera notée par

$$
\alpha_{0} /(j \cdot \omega)
$$

et le déplacement par

$$
\alpha_{0} /(j \cdot \omega)^{2} \text { ou }-\alpha_{0} / \omega^{2}
$$

De même l'accélération sismique verticale sera désignée par $\gamma_{0}$

Les composantes des accélération de l'eau dans le réservoir seront désignées $\operatorname{par} \alpha(x, z)$ et $\gamma(x, z)$. Contrairement à $\alpha_{0}$ et $\gamma_{0}$ ce ne sont pas des constantes mais des fonctions des coordonnées $x$ et $z$. Nous ne considérerons que les fluctuations de pression $P$ autour de la pression hydrostatique, en fait, nous n'utiliserons que la quantité $Q(x, z)=P / \rho, \rho$ étant la masse spécifique de l'eau.

Nous désignerons par $a(x, z)$ les déplacements du parement amont par rapport à la position d'équilibre hydrostatique.

Les équations du mouvement de l'eau sont classiques et peuvent s'écrire

$$
\begin{gathered}
\Delta Q+k^{2} \cdot Q=0 \\
\alpha=-\frac{\partial Q}{\partial x} \\
\gamma=-\frac{\partial Q}{\partial z}
\end{gathered}
$$

où l'on a posé $k=\omega / c, c$ étant la vitesse de propagation des ondes de pression dans l'eau ( $1500 \mathrm{~m} / \mathrm{s}$ environ).

Nous prendrons $Q$ comme inconnue, les équations 5 et 6 permettant d'en déduire les accélérations et d'exprimer les conditions aux limites. Par exemple, pour exprimer les équations des mouvements élastiques de la protection pneumatique, nous utiliserons systématiquement les relations des types 2 et 3 nous obtiendrons

$$
\begin{aligned}
&-\rho \cdot Q-f \cdot\left(j \cdot \omega \cdot a-\alpha_{0} /(j \cdot \omega)\right) \\
&-r \cdot\left(a+\alpha_{0} / \omega^{2}\right)=0 \\
& \text { pour } \quad x=0 \text { et }-H<z<0
\end{aligned}
$$

L'accélération horizontale de l'eau, sur le parement amont, est égale à $-\omega^{2} \cdot a$ donc, compte tenu de (5) et après quelques transformations, on peut écrire la condition aux limites sur ce parement.

$$
\begin{aligned}
& \rho \cdot Q+\left(r / \omega^{2}-f /(j \cdot \omega)\right) \frac{\partial Q}{\partial x} \\
&=\left(f /(j \cdot \omega)-r / \omega^{2}\right) \cdot \alpha_{0} \\
& \text { pour } \quad x=0 \text { et }-H<z<0
\end{aligned}
$$

Les autres conditions aux limites sont simples

$$
\begin{array}{cl}
\frac{\partial Q}{\partial z}=-\gamma_{0} & \text { pour } z=-H \\
Q=0 & \text { pour } z=0
\end{array}
$$

En toute rigueur, il faut y ajouter une condition de " rayonnement " (pas d'ondes venant de l'infini amont) et une condition de non infinité à l'infini amont.

\subsection{Forme de la solution}

La solution $Q$ peut être mise sous la forme

$Q=\sum Q_{n}^{\prime} \cdot \sin \left(r_{n} \cdot z\right)$.

$$
\operatorname{EXP}\left(-q_{n} \cdot x\right)-\gamma_{0} \cdot \sin (k \cdot z) /(k \cdot \cos (k \cdot H))
$$

où $Q_{n}^{\prime}$ sont des coefficients à déterminer $(n=1,2,3 \ldots)$

$$
\begin{aligned}
& r_{n}=(2 \cdot n-1) \cdot \pi /(2 \cdot H) \\
& q_{n}=\sqrt{r_{n}^{2}-\mathrm{k}^{2}} \quad \text { si } r_{n}>k \\
& q_{n}=j \cdot \sqrt{k^{2}-r_{n}^{2}} \quad \text { si } r_{n}<k
\end{aligned}
$$

Notons que nous excluons provisoirement les cas où $k$ est égal à une des valeurs de $r_{n}(\cos (k \cdot H)=0)$.

Il est facile de vérifier que la fonction $Q$ ainsi définie satisfait à l'équation générale 4 , aux conditions aux limites 9 et 10 ainsi qu'aux conditions à l'infini et de rayonnement. Il reste à déterminer les fonctions $Q_{n}^{\prime}$ pour satisfaire à la condition 8 .

On déduit facilement de 11 la valeur de $Q$ pour $x=0$ en remplaçant $\sin (k \cdot z)$ par son développement en somme de fonctions $\sin \left(r_{n} \cdot z\right)$ dans l'intervalle $(-H, 0)$. On peut alors écrire

$$
Q=\sum Q_{n} \cdot \sin \left(r_{n} \cdot z\right) \text { pour } x=0
$$

où

$$
Q_{n}=Q_{n}^{\prime}+(-1)^{n} \cdot 2 \cdot \gamma_{0} /\left(H \cdot q_{n}^{2}\right)
$$

On a, également pour $x=0$

$$
\begin{aligned}
& \frac{\partial Q}{\partial x}=-\sum Q_{n}^{\prime} \cdot q_{n} \cdot \sin \left(r_{n} \cdot z\right) \\
& =\sum\left((-1)^{n} \cdot 2 \cdot \gamma_{0} /\left(H \cdot \mathrm{q}_{n}\right)\right. \\
& \left.\quad-Q_{n} \cdot q_{n}\right) \cdot \sin \left(r_{n} \cdot z\right)
\end{aligned}
$$

En substituant ces expressions dans 8 et en remplaçant la constante $\alpha_{0}$ par son développement en somme de fonctions $\sin \left(r_{n} \cdot z\right)$, on obtient

$\sum\left[\rho \cdot Q_{n}+\left(r / \omega^{2}-f /(j \cdot \omega)\right)\right.$

$\left.\cdot\left((-1)^{n} \cdot 2 \cdot \gamma_{0} /\left(H \cdot q_{n}\right)-Q_{n} \cdot q_{n}\right)\right] \cdot \sin \left(r_{n} \cdot z\right)$

$=\left(f /(j \cdot \omega)-r / \omega^{2}\right) \cdot \alpha_{0}=\sum\left(r / \omega^{2}\right.$

$\left.f /(j \cdot \omega)) \cdot\left(2 \cdot \alpha_{0} / r_{n} \cdot H\right)\right) \cdot \sin \left(r_{n} \cdot z\right)$

D'où l'on déduit les $Q_{n}$

$$
\begin{aligned}
& Q_{n}=2 \cdot\left[(r+j \cdot \omega \cdot f) \cdot \alpha_{0} / r_{n}-\right. \\
& \begin{array}{r}
\left.(r+j \cdot \omega \cdot f) \cdot(-1)^{n} \cdot \gamma_{0} / q_{n}\right] /\left[H \cdot \omega^{2} \cdot \rho\right. \\
-H \cdot q_{n} \cdot(r+j \cdot \omega \cdot f)
\end{array}
\end{aligned}
$$

\subsection{Formules "classiques" sans protection pneumatique}

Il suffit de considérer que la rigidité $r$ devient infinie, on a

$$
\begin{aligned}
Q_{n}=-2 \cdot \alpha_{0} /\left(H \cdot r_{n} \cdot q_{n}\right) \\
+(-1)^{n} \cdot 2 \cdot \gamma_{0} /\left(H \cdot q_{n}^{2}\right)
\end{aligned}
$$




\subsection{Résonances}

Il y a résonance lorsque la fréquence $\omega$ est telle que l'une des valeurs $q_{n}$ est nulle. Par exemple, $q_{1}=0$ correspond au cas d'une résonance verticale en "quart d'onde " du réservoir. Pour que les formules conservent un sens physique, il serait nécessaire de tenir compte des pertes d'énergie dans les mouvements oscillatoires. Ces pertes d'énergie sont relativement faibles, ce qui signifie que les accroissements de réponse dynamique seront, non pas infinis, mais très considérables au voisinage des fréquences de résonance. C'est notamment à cause de celà que les formules de Westergaard donnent des valeurs sous-estimées des surpressions hydrodynamiques, ainsi que nous l'avons indiqué plus haut.

Sans entrer dans le détail de calculs tenant compte des pertes d'énergie, on peut remarquer que $q_{n}$ figure au dénominateur des deux termes de 19. Cela signifie que les séismes horizontaux comme les séismes verticaux donnent lieu à des phénomènes de résonance en l'absence de protection. Au contraire, avec celle-ci, les résonances disparaissent complètement pour les séismes horizontaux et sont très atténuées pour les séismes verticaux, $q_{n}^{2}$ étant remplacé par $q_{n}$ au dénominateur du terme correspondant.

\subsection{Poussées et couples de renversement}

On calcule facilement, par intégration sur l'axe vertical, la poussée (par unité de largeur) correspondant à la nième composante

$$
\rho \cdot Q_{n} / r_{n}
$$

et le couple de renversement par unité de largeur

$$
-\rho \cdot Q_{n}\left(H / r_{n}+(-1)^{n} / r_{n}^{2}\right)
$$

